\title{
Metaheuristic optimization algorithms for the optimal coordination of plug-in electric vehicle charging in distribution systems with distributed generation
}

\author{
Nataly Bañol Arias ${ }^{a}$, John F. Franco ${ }^{a}$, Marina Lavorato ${ }^{\mathrm{b}, *}$, Rubén Romero ${ }^{\mathrm{a}}$ \\ a UNESP - Universidade Estadual Paulista, Faculdade de Engenharia de Ilha Solteira, Departamento de Engenharia Elétrica, Ilha Solteira, São Paulo, Brazil \\ b PUC -Campinas - Pontifícia Universidade Católica de Campinas, Faculdade de Engenharia Elétrica, Campinas, Sao Paulo, Brazil.
}

\section{A R T I C L E I N F O}

\section{Article history:}

Received 20 May 2016

Received in revised form

14 September 2016

Accepted 18 September 2016

Available online 20 October 2016

\section{Keywords:}

Electrical distribution system

Hybrid algorithm

Metaheuristic

Plug-in electric vehicle charging

coordination

\begin{abstract}
A B S T R A C T
This paper proposes three metaheuristic optimization techniques to solve the plug-in electric vehicle (PEV) charging coordination problem in electrical distribution systems (EDSs). Optimization algorithms based on tabu search, greedy randomized adaptive search procedure, and a novel hybrid optimization algorithm are developed with the objective of minimizing the total operational costs of the EDS, considering the impact of charging the electric vehicle batteries during a specific time period. The proposed methodologies determine a charging schedule for the electric vehicle batteries considering priorities according to the PEV owners charging preferences. A 449-node system with two distributed generation units was used to demonstrate the efficiency of the proposed methodologies, taking into account different PEV penetration levels. The results show that the charging schedule found makes the economic operation of the EDS possible, while satisfying operational and priority constraints.
\end{abstract}

(c) 2016 Elsevier B.V. All rights reserved.

\section{Introduction}

The future large-scale penetration of plug-in electric vehicles (PEVs) will bring both positive and negative consequences, depending on different points of view [1]. In the environmental context, PEVs reduce greenhouse gas emissions $\left(\mathrm{CO}_{2}, \mathrm{SO}_{2}\right.$, and $\left.\mathrm{NO}_{x}\right)$ by decreasing fossil fuel consumption [2]. On the other hand, PEVs benefit the transportation sector by providing low operational costs [3]. However, from the point of view of the electrical distribution system (EDS) operation, PEVs represent a significant new load that must be supplied efficiently by the system, fulfilling the final consumers needs [1]. Previous works have shown that the EDS operation is strongly affected when PEV charging is not properly coordinated [4]. Several problems, such as higher load peak, decrease in service quality, degradation of the voltage profile, overload of circuits, and increase in energy losses are consequences of PEV penetration [4-6]. Nevertheless, those impacts can be mitigated by using heuristic procedures to solve the PEV charging

\footnotetext{
* Corresponding author at: PUC -Campinas - Pontifícia Universidade Católica de Campinas, Faculdade de Engenharia Elétrica, Campinas, Sao Paulo, Brazil.

E-mail addresses: natycanta@gmail.com

(N.B. Arias), jffranco@gmail.com (J.F. Franco), mlavorato@gmail.com (M. Lavorato), ruben@dee.feis.unesp.br (R. Romero).
}

coordination (PEVCC) problem [4,7-10]. Charging strategies based on quadratic optimization models $[6,11]$ and mixed-integer linear programming (MILP) $[12,13]$ have been proposed. An MILP approach that minimizes the total daily cost due to EV charging to define the charging schedule in real-time was proposed in [13]. Other approaches based on decentralized PEV charging strategies have been investigated in [13-15]. In these latter works, each PEV is allowed to determine its own charging pattern, i.e., there is no central operator deciding when and at what rate each individual PEV will be charged.

On the other hand, centralized approaches have been considered for the PEVCC problem $[10,16,17]$. An algorithm for real-time smart load management applied to the PEVCC problem, which minimizes the total generation cost and the energy losses of the network, was developed in [10]. Furthermore, an evolutionary algorithm [16] and a metaheuristic method based on particle swarm optimization, genetic algorithms, and simulated annealing [17] were developed in order to provide quality solutions for the PEVCC problem.

The operation of the EDS, considering PEV charging and integration of DG sources, is a challenge for the EDS operator. Some authors have addressed this subject [8,10,18,19]. Ref. [18] proposed an online fuzzy coordination algorithm for the PEVCC problem that minimizes the total cost of energy generation and power losses, while maintaining the network's operational constraints and considering priority charging and DG resources. An optimization 
methodology for designing integrated PEV charging systems with multiple chargers, renewable DG, and storage units was proposed in [19].

Some other studies have considered the relationship between PEV charging and photovoltaic (PV) generation [20-22], as well as the relationship between PEV charging and wind power generation $[23,24]$. Likewise, the PEVCC problem, including DG operation in a three-phase generic LV distribution network, was investigated in [25]; nevertheless, control of the DG units was not considered. The management of DG units, demand response, and electric vehicles with V2G technology in a smart grid environment were considered in an optimization approach based on simulating annealing (SA) in [26]; additionally, a methodology based on mixed-integer nonlinear programming (MINLP) was proposed to compare the results obtained with the SA approach, showing an important reduction in the computational times.

\subsection{Contributions}

Classical optimization techniques can be used to solve the PEVCC problem. However, in practice mathematical formulations for this problem, such as MINLP, are affected by scalability and convergence issues, due to the non-convex nature of the problem. The computational effort of the algorithms (e.g., non-linear branch and bound) used to solve this kind of problem is directly related to the number of binary variables. For the PEVCC problem, this number is proportional to the number of PEVs in the EDS. Therefore, the computational effort to solve the PEVCC problem increases with the penetration level of PEVs, as well as with the size of the network, making it hard to solve using classical optimization techniques. For this reason, the application of alternative optimization techniques, such as metaheuristics, should be investigated [18]. This work proposes novel optimization algorithms based on the metaheuristics Tabu Search (TS) [27] and Greedy Randomized Adaptive Search Procedure (GRASP) [28], to minimize the total operational costs of the EDS by finding the best possible PEV charging schedule that satisfies the PEV energy requirements, the priority charging conditions of the PEVs, and the operational constraints of the EDS. Also, a hybrid algorithm that, improves on the solutions found using the classical TS and GRASP algorithms is proposed. The performance of the proposed optimization methods was investigated in a 449-node EDS including medium and low voltage feeders.

The main contributions of this paper are as follows:

1. Novel metaheuristic algorithms for solving the PEVCC problem in EDSs, considering DG units, as well as the electrical and operational constraints of the system;

2. A procedure to obtain a suitable charging schedule for the PEVs with efficient computational behavior, which uses a sensitivity index for the variation of the energy costs related to the PEV charging.

\section{Mathematical formulation of the PEVCC problem}

The PEVCC problem can be formulated as an MINLP model in which the steady-state operation of the EDS is modeled based on $[29,30]$. The solution of this model provides the optimal charging schedule for the PEV batteries. In order to model the PEVCC problem, the following assumptions are considered:

1. The PEV batteries must be charged in a defined time period divided in time intervals, in which the charging process is executed;

2. The energy required by each PEV battery is known at the beginning of the time period;
3. The EDS operator controls the PEV charging process, i.e., the PEVs are equipped with communication devices that make it possible to control their charging state in each time interval;

4. Additionally, in each state of the charging schedule the operational constraints of the EDS must be satisfied.

\subsection{Objective function}

The main objective of the PEVCC problem is to minimize the total operational cost of the EDS (1). The objective function proposed in this work is formed by three components, calculated over the time period as the sum of the cost of the energy supplied by the substation and the DG units (2); a term that encourages the charging of PEVs with priority conditions (3); and the penalization costs associated with voltage limit violations, current limit violations, and non-charged PEV energy (4). The cost represented in (3) enables PEV charging with priority conditions, where $\rho_{t}$ is a decreasing parameter that can be calculated by using the cost function defined by the equation $2^{[6+24 H(t-18)-t]}$, and $H(t)$ is a Heaviside function. This term encourages the charging of priority PEVs as soon as possible over the charging of non-priority PEVs.

$\min \beta+\gamma+\delta$

where

$$
\begin{aligned}
& \beta=\sum_{t \in T} \mu_{t} \Delta_{t} P_{S E, t}^{g}+\eta \sum_{t \in T} \Delta_{t} \sum_{j \in N} P_{j, t}^{g} \quad \forall j \in N, \forall t \in T \\
& \gamma=-\sum_{j \in N} \sum_{t \in T} \kappa_{j} \rho_{t} P_{j, t}^{v} \quad \forall j \in N, \forall t \in T \\
& \delta=\theta \sum_{j \in N} \max \left[\left(\underline{V}-V_{j}\right), 0\right]^{2}+\sigma \sum_{i j \in L} \max \left[\left(I_{i j}-\bar{I}_{i j}\right), 0\right]^{2}+\varphi \sum_{j \in N} \pi_{j}
\end{aligned}
$$

A solution is only feasible when the penalizations related to the violation of voltage and current limits in (4) are equal to zero, which guarantees a feasible operation. Furthermore, the terms related to the PEVs in (3) and (4) indicate the charging preferences and the level of satisfaction of the PEV owners. Therefore, it is expected that all PEVs with priority charging conditions will be charged as soon as possible and that their energy requirements will be totally fulfilled, given that the system operates without limit violations.

\subsection{Network constraints}

Three types of constraints are considered in the proposed model:

- Active and reactive power balance constraints along with the voltage drop in the circuits, which represent Kirchhoffs laws and make it possible to calculate the steady-state operation of the EDS.

$$
\begin{aligned}
& \sum_{i j \in L} P_{i j, t}-\sum_{j k \in L}\left(P_{j k, t}+R_{j k, t} I_{j k, t}^{2}\right)+P_{j, t}^{g}=P_{j, t}^{d}+P_{j, t}^{v} \quad \forall j \in N, \forall t \in T \\
& \sum_{i j \in L} Q_{i j, t}-\sum_{j k \in L}\left(Q_{j k, t}+X_{j k, t} I_{j k, t}^{2}\right)+Q_{j, t}^{g}=Q_{j, t}^{d} \quad \forall j \in N, \forall t \in T \\
& V_{j, t}^{2}-V_{k, t}^{2}=2\left(R_{j k} P_{j k, t}+X_{j k} Q_{j k, t}\right)+Z_{j k}^{2} I_{j k, t}^{2} \quad \forall i j \in L, \forall t \in T \\
& V_{k, t}^{2} I_{j k, t}^{2}=P_{j k, t}^{2}+Q_{j k, t}^{2} \quad \forall j k \in L, \forall t \in T
\end{aligned}
$$

Eqs. (5) and (6) represent the active and reactive power balance and guarantee that all loads are supplied. Eqs. (7) and (8) represent the application of Kirchhoff's voltage law [31]. The set of Eqs. (5)-(8) is used to represent the steady-state operation of 
the EDS, assuming a radial operation of the network [32]. Nevertheless, the operation of weakly meshed networks can be represented by including additional constraints related to the voltage phase angle; alternatively, the set of Eqs. (5)-(8) can be replaced by the standard AC power injection equations.

- Operational constraints such as voltage limits, current capacity, and DG limits.

$0 \leq P_{j, t}^{g} \leq \bar{P}_{j}^{g} \quad \forall j \in N, t \in T$

$Q_{j}^{g} \leq Q_{j, t}^{g} \leq \bar{Q}_{j}^{g} \quad \forall j \in N, t \in T$

$\left|Q_{j, t}^{g}\right| \leq P_{j, t}^{g} \tan \left(\operatorname{arcos}\left(p f_{j}\right)\right) \quad \forall j \in N, t \in T$

Constraints (9)-(11) represent the operational limits of the DG units, taking into account that these constraints only appear for nodes with DG, and not substation nodes. The voltage limits and current capacity constraints are not presented individually because they are treated as penalizations in the objective function (1).

- Constraints associated with the PEVs: energy balance in the batteries, PEV maximum power consumption, and priority conditions.

$\pi_{j}=E_{j}^{v}-S O C_{j}^{i n i}-\varepsilon_{j}^{c h} \sum_{t \in T} \Delta_{t} P_{j, t}^{v} \quad \forall j \in N$

$P_{j, t}^{v}=\bar{P}^{v} x_{j, t}^{v} \quad \forall j \in N, t \in T$

Eq. (12) represents the energy balance of a PEV battery, which is expressed in terms of the energy not charged in the battery at the end of the time period $\left(\pi_{j}\right)$, the energy required by the battery $\left(E_{j}^{v}\right)$, the initial state of charge of the PEV battery $\left(S O C_{j}^{i n i}\right)$, and the total power demanded $\left(P_{j, t}^{v}\right)$ affected by the efficiency of the PEV battery charger $\left(\varepsilon_{j}^{c h}\right)$. This equation allows for the calculation of $\pi_{j}$, which penalizes the objective function when $\pi_{j}>0$, i.e., when there is energy not charged in the PEV battery. Eq. (13) determines the power demanded by a PEV, according to the maximum power of the PEV battery and its charging state (where $x_{j, t}^{v}$ is 1 if the battery has been charged at time interval $t$; otherwise, $x_{j, t}^{v}$ is 0 ).

The optimization model, (1)-(13), is an MINLP, due to the square terms in (4)-(8), as well as the product of variables in (8). This kind of model can be solved using different techniques, as presented in [30], in order to solve the problem more easily. Nevertheless, classical optimization techniques could present performance issues when the problem has large dimensions. As a result, it might be difficult to solve the problem in reasonable computational time. On the other hand, heuristic and metaheuristic techniques can provide good-quality solutions that are close to the optimal solution or even the optimal solution with less computational effort, which makes them suitable for solving the PEVCC problem.

\section{Metaheuristic optimization algorithms for the PEV charging coordination problem}

New optimization algorithms based on TS and GRASP are presented in this section to solve the PEVCC problem. These algorithms have been developed to solve the PEVCC problem in EDSs and to minimize the total operational costs defined by (1). Additionally, a hybrid algorithm that combines TS and GRASP is developed.

In order to assess the steady-state operation of the EDS, including PEV charging and DG source operation, a specialized radial load flow based on the backward/forward load flow proposed in [32] is used. All loads are represented as constant active and reactive power loads.

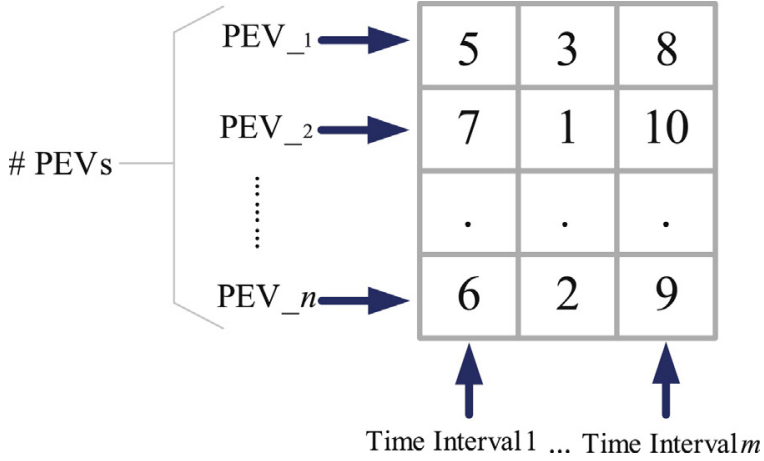

Fig. 1. Example of the proposed codification.

\subsection{Tabu search algorithm for the PEVCC}

As any local search method, TS employs four basic components [30]:

1. A codification structure that represents a solution proposal and defines the search space;

2. A procedure to generate an initial solution;

3. A neighborhood structure to generate a subset of solutions;

4. A transition mechanism that establishes how to pass from a solution proposal to a new solution.

\subsubsection{Codification structure}

A solution proposal is represented in a matrix in which each row corresponds to a PEV and the number of columns is defined as the maximum number of time intervals required to charge the PEV batteries, as given by (14).

$\lambda=\frac{E_{j}^{v}}{\bar{P}^{v} \Delta_{t}}$

As illustrated in Fig. 1, the elements of the codification matrix are integer numbers that belong to the set of time intervals $T$ and represent the time interval in which the PEV batteries are charged. In this way, the number 1 represents the time interval between 18:00 and 19:00, the number 2 represents the time interval between 19:00 and 20:00, and so on. For a given PEV, the chosen codification indicates the time intervals in which the PEV battery is charged; however, it does not necessarily indicate the sequence in which the PEV battery is charged. Fig. 1 illustrates the case in which three time intervals are needed to charge the PEVs $(\lambda=3)$.

\subsubsection{Initial solution}

As the energy costs could change over the time intervals, an initial solution can be built by charging the PEV batteries without priority conditions during the time intervals with the lower energy costs. In this case, usually the chosen time intervals are in the early morning. On the other hand, the charging of PEVs with priority conditions should be carried out during the first hours of the time period because it is expected that these PEVs need to be charged as soon as possible. So, following these conditions, the initial solution is easy to be generated. However, voltage and current limit violations may appear due to the simultaneous charging of the PEVs. If necessary, the optimization process can search in the solution space to eliminate such unfeasibilities and find a good-quality solution. The proposed initial solution simulates an uncoordinated charging process in which the PEVs are connected to the network without being managed by the EDS operator. The proposed methodology allows the EDS operator to identify suitable time intervals to charge the PEVs. 


\subsubsection{Neighborhood structure}

For a given solution proposal, a neighbor solution can be generated by changing the time intervals stored in one position of the codification matrix. That operation can be carried out for each row of the matrix (associated with a PEV) and for each column (associated with a charging time interval), with the condition that the new generated time interval differ from the current time intervals present in the selected row. Additionally, the new time interval must belong to the set of time intervals $T$. Thus, the feasibility of the new solution proposal is maintained from the point of view of the codification.

\subsubsection{Transition mechanism}

After evaluating all the neighbor solutions for a current configuration, a solution is chosen by applying the TS criteria and using a short-term memory structure, as explained in [27].

\subsubsection{Steps of the tabu search algorithm}

Step 1: Generate the initial solution.

Step 2: Evaluate the neighborhood.

Step 3: Select the solution according to the TS transition mechanism.

Step 4: Update the TS short-term memory structure.

Step 5: Verify the stopping criterion. If it is satisfied, stop. Otherwise, return to Step 2 .

\subsection{GRASP algorithm for the PEVCC}

The GRASP algorithm is used to solve the PEVCC problem by adapting its construction and local search phases [28]. The codification of the solution proposal is presented in Section 3.1.1.

\subsubsection{Construction phase}

In the construction phase, an initial solution is iteratively formed by selecting one element at a time. The construction phase begins with an empty initial solution and, in each iteration, a PEV is chosen to be charged in a specific time interval. The process stops when $\lambda$ time intervals have been assigned to all PEVs, i.e., all the periods required to charge the battery of each PEV in the system have been assigned. In each iteration of the construction phase, the next element to be added is selected from a Restrict Candidates List (RCL), which is built using a sensitivity index (15) and parameter $\alpha \in[0$, 1]. The sensitivity index $\Phi_{k, t}$ estimates, in each iteration, the variation of the objective function when the charging of a new element is considered, e.g., the $k$ th PEV at the $t$ th time interval. The use of this index to evaluate the objective function avoids the calculation of a complete load flow for all possible candidates.

$\Phi_{k, t}=\mu_{t} \Delta_{t} \Delta P_{k, t}^{L o s s}+\mu_{t} \Delta_{t} \bar{P}^{v}-\kappa_{n} \rho_{t} P_{k, t}^{v}$

Thus, the sensitivity index improves the efficiency of the algorithm by reducing required computational effort. Using the sensitivity index, the RCL is defined by (16).

$R C L=\left\{k, t \in X \mid \Phi_{\min } \leq \Phi_{k, t} \leq \Phi_{\min }+\alpha\left(\Phi_{\max }-\Phi_{\min }\right)\right\}$

Where $X$ is the set of index that represent the elements allowed to be added to the solution. $\Phi_{\min }$ and $\Phi_{\max }$ are the minimum and the maximum values for the index. The objective of (16) is to select the elements with the lowest values of $\Phi_{k, t}$. The value of $\alpha$ is adjusted experimentally by doing simulations with different values.

When the RCL is created, an element is chosen using a linear probability distribution function described in (17), where $r_{i}$ represents the position of element $i$ in the sorted RCL, according to the quality of $\Phi_{k, t}$. Therefore, the probability of choosing element $i$ is found using (18).

$b_{i}=\frac{1}{r_{i}}$

$p_{i}=\frac{b_{i}}{\sum_{j \in R C L} b_{j}}$

The selection of the ith element is implemented using a random number generator and a proportional selection criterion. The selected element is added to the solution in construction, and then, the objective function is calculated again using (1). This iterative process ends when a solution has been completely constructed. The construction phase of the proposed GRASP algorithm is comprised of the following steps:

Step 1: Read the input data and set $\alpha$.

Step 2: Define an empty set as the initial solution, i.e., the EDS with no PEVs connected to the network. Calculate the steady-state operation of the EDS without PEVs.

Step 3: Calculate $\Phi_{k, t}$ using (15) for all the PEVs in all time intervals $t \in T$, which have not yet been added to the solution.

Step 4: Create the RCL using (16).

Step 5: Calculate $p_{i}$ for all elements of the RCL and choose the element that will be added to the solution in construction.

Step 6: Evaluate the objective function.

Step 7: Check the stopping criterion. If it is satisfied, stop. Otherwise, return to step 3 .

\subsubsection{Local search phase}

The main objective of the local search phase is to improve the solution obtained in the construction phase. A steep descent heuristic algorithm is implemented using the neighborhood structure proposed in Section 3.1.3. After all the neighbors have been evaluated, the next solution will be the best neighbor solution found.

The local improvement process ends when none of the neighbor solutions are better than the current solution. The local search phase of the proposed GRASP algorithm has the following steps:

Step 1: Read the current solution found in the constructive phase of the GRASP algorithm.

Step 2: Generate a neighbor solution.

Step 3: Evaluate the objective function.

Step 4: If the neighbor solution is better than the current solution, make the transition to the neighbor solution.

Step 5: Check the stopping criterion. If there is no neighbor solution better than the current one, then the local search phase is finished. Otherwise, return to step 2.

\subsubsection{Stopping criterion of the GRASP algorithm}

The stopping criterion established in the GRASP algorithm is a maximum execution time. At the end of the process, when the maximum time is reached, the best found solution is the solution for the PEVCC problem.

\subsection{Hybrid optimization algorithm (HOA)}

Hybrid algorithms are frequently used to obtain good solutions for a certain problem by combining different search strategies from two or more methodologies in the solution space [34]. They are developed in order to improve the solutions found using simple algorithms. In this work, a novel hybrid optimization algorithm is proposed in order to improve the found solutions via the TS and GRASP methodologies previously presented. 


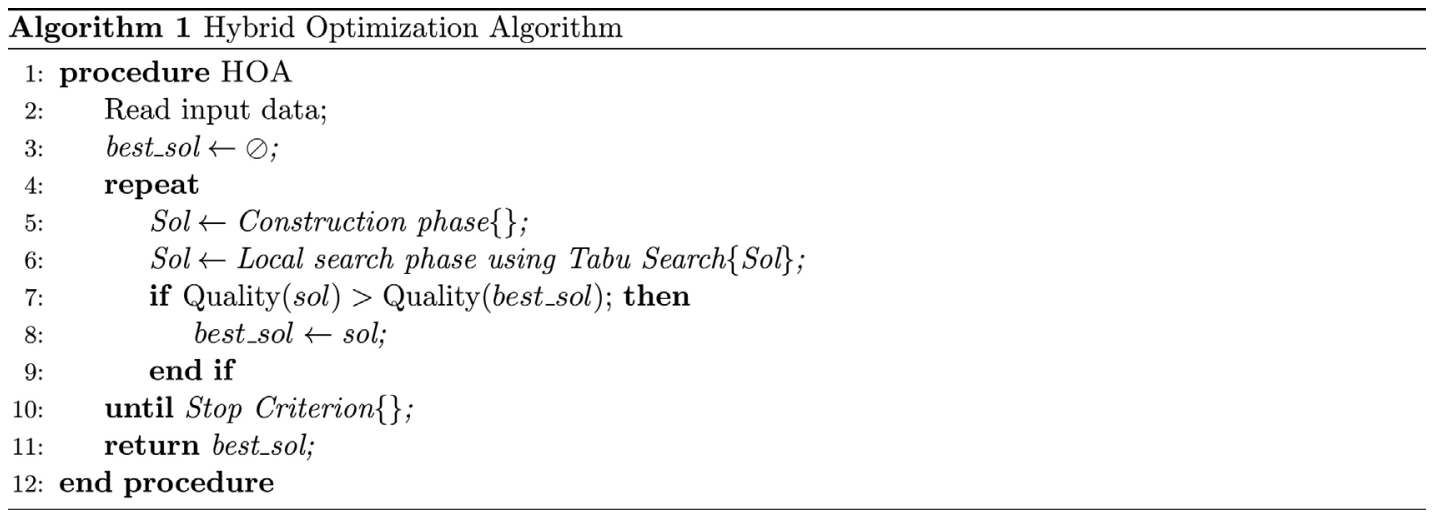

Fig. 2. Pseudocode of the proposed hybrid optimization algorithm.

Table 1

PEV charging scenarios.

\begin{tabular}{lcc}
\hline Cases & Priority & DG \\
\hline A & $\sqrt{ }$ & - \\
B & $\sqrt{ }$ & $\sqrt{ }$ \\
C & - & - \\
D & - & $\sqrt{ }$ \\
\hline
\end{tabular}

The HOA takes advantage of the TS and GRASP characteristics. This way, the proposed HOA uses the GRASP's construction phase to generate a good-quality initial solution, and then the short-term strategies of the TS algorithm are applied in the local search phase. The HOA is an iterative process that, can be executed in a limited amount of time. Due to the features of GRASP, a different initial solution is generated in each iteration, making it possible to diversify the search process by exploring different regions of the search space. A pseudocode of the proposed hybrid optimization algorithm is shown in Fig. 2.

One advantage of the proposed HOA is its simple and practical application. Furthermore, the results demonstrate that the proposed HOA has better performance than the TS and GRASP methodologies, as shown in the results section.

\subsection{PEV charging and DG units}

As mentioned in [35], DG units enhance the performance of the system, improving the voltage profiles and reducing the active power losses and total operational cost. Therefore, DG units were included in the optimization methodologies in order to identify their impact on the EDS operation and the scheduling of the PEV battery charging. Each methodology (TS, GRASP, and HOA) is used to define the optimal power injected by the DG units in each time interval. This optimization process consists of a unidimensional search, which makes small variations in the DG unit power, starting with an initial value, in order to improve the objective function.

\section{Test and results}

The algorithms were developed in the programming language $\mathrm{C}++$ and were executed using a Dell PowerEdge R910x64 computer with six processors at $1.87 \mathrm{HGz}$ Model i7 4807. A 449-node distribution system was used to compare the performance of the TS, GRASP, and HOA methods. Four PEV charging scenarios, as shown in Table 1, were taken into account. In these scenarios, two PEV penetration levels were considered: 47\% (198 PEVs) and 63\% (264 PEVs). The number of binary variables for all cases was calculated as 2772 and 3696 for $47 \%$ and $63 \%$ PEV penetration, respectively. Additionally, it was assumed that some of the PEVs should be charged with priority. The 449-node system, whose data are available in [10], consisted of a network with 31 medium-voltage nodes $(23 \mathrm{kV})$ and 22 low-voltage feeders ( $415 \mathrm{~V}$ ), each with 19 nodes. The PEVs were connected in the low-voltage network.

The energy cost and the load for each time interval are available in [30]. The considered time period for the charging spanned from 18:00 h until 08:00 h, broken down into time intervals of $1 \mathrm{~h}$, i.e., $\Delta_{t}=1 \mathrm{~h}$. Therefore, time interval $1 \mathrm{was}$ from $18: 00 \mathrm{~h}$ to $19: 00 \mathrm{~h}$, time interval 2 was from 19:00 h to 20:00 h, and so on. The voltage limits for all cases were $V=0.9 \mathrm{pu}$ and $\bar{V}=1.0 \mathrm{pu}$, while the maximum current for all the low-voltage feeders was $200 \mathrm{~A}$.

There were two DG units connected at nodes 14 and 17, corresponding to a DG penetration of about $40 \%$ of the maximum demand. One of the DG units was a small hydro power plant with a fixed generation in each time interval. The other was a DG unit whose generation can vary over time depending on system requirements. The cost of the energy supplied by both DG units $(\eta)$ was $0.04 \mathrm{US} \$ / \mathrm{kWh}$. The active and reactive power limits were $\bar{P}_{n}^{g}=200 \mathrm{~kW}, \bar{Q}_{n}^{g}=100 \mathrm{kVAr}$, and $\underline{Q}_{n}^{g}=$ $-100 \mathrm{kVAr}$ with a fixed power factor equal to 0.9 . The capacity of the PEV batteries was $20 \mathrm{kWh}$, according to information provided in [33]. The maximum power of the battery was $4 \mathrm{~kW}$ as assumed in [10] and the battery charger efficiency was assumed as $100 \%$.

Table 2

Comparative table of the objective function in each PEV charging scenario.

\begin{tabular}{|c|c|c|c|c|c|c|c|c|c|c|c|c|c|}
\hline \multirow{2}{*}{\multicolumn{2}{|c|}{$\begin{array}{l}\text { Time limit } \\
\text { Case }\end{array}$}} & \multicolumn{3}{|l|}{$300 \mathrm{~s}$} & \multicolumn{3}{|l|}{$600 \mathrm{~s}$} & \multicolumn{3}{|l|}{$1200 \mathrm{~s}$} & \multicolumn{3}{|l|}{$4 \mathrm{~h}$} \\
\hline & & Grasp & Tabu & Hybrid & Grasp & Tabu & Hybrid & Grasp & Tabu & Hybrid & Grasp & Tabu & Hybrid \\
\hline \multirow{4}{*}{$47 \%$} & $\mathrm{~A}$ & -1540.67 & -1540.67 & -1540.67 & -1540.67 & -1540.67 & -1540.67 & -1540.67 & -1540.67 & -1540.67 & -1540.67 & -1540.67 & -1540.67 \\
\hline & B & -1616.62 & -1614.93 & -1616.28 & -1616.28 & -1614.93 & -1616.62 & -1616.62 & -1614.93 & -1616.62 & -1615.69 & -1614.93 & -1617.30 \\
\hline & C & 515.12 & 515.47 & 514.85 & 514.90 & 515.17 & 514.59 & 515.23 & 514.62 & 514.59 & 515.02 & 514.62 & 514.59 \\
\hline & $\mathrm{D}$ & 449.12 & 450.69 & 448.90 & 448.70 & 450.69 & 448.53 & 449.23 & 450.69 & 448.47 & 449.47 & 450.69 & 448.30 \\
\hline \multirow{4}{*}{$63 \%$} & $\mathrm{~A}$ & -2856.42 & -2856.76 & -2856.92 & -2857.30 & -2856.76 & -2857.34 & -2857.33 & -2856.76 & -2857.34 & -2857.34 & -2856.76 & -2857.34 \\
\hline & B & -2926.39 & -2926.43 & -2927.51 & -2927.05 & -2926.43 & -2927.61 & -2927.38 & -2926.43 & -2927.72 & -2927.05 & -2926.43 & -2927.72 \\
\hline & $\mathrm{C}$ & 557.82 & 2264.29 & 557.41 & 556.99 & 557.93 & 556.87 & 557.04 & 556.95 & 556.71 & 557.05 & 556.95 & 556.58 \\
\hline & $\mathrm{D}$ & 492.35 & 493.73 & 491.98 & 492.06 & 493.73 & 491.78 & 492.10 & 493.17 & 491.35 & 491.93 & 491.62 & 491.28 \\
\hline
\end{tabular}


Table 3

Percentage deviation of the objective function in each PEV charging scenario.

\begin{tabular}{|c|c|c|c|c|c|c|c|c|c|c|}
\hline \multirow{2}{*}{\multicolumn{2}{|c|}{$\begin{array}{l}\text { Time limit } \\
\text { Case }\end{array}$}} & \multicolumn{3}{|l|}{$300 s$} & \multicolumn{3}{|l|}{$600 s$} & \multicolumn{3}{|l|}{$1200 s$} \\
\hline & & Grasp & Tabu & Hybrid & Grasp & Tabu & Hybrid & Grasp & Tabu & Hybrid \\
\hline \multirow{4}{*}{$47 \%$} & A & 0.00 & 0.00 & 0.00 & 0.00 & 0.00 & 0.00 & 0.00 & 0.00 & 0.00 \\
\hline & B & 0.00 & 0.10 & 0.02 & 0.02 & 0.10 & 0.00 & 0.00 & 0.10 & 0.00 \\
\hline & $\mathrm{C}$ & 0.10 & 0.17 & 0.05 & 0.06 & 0.11 & 0.00 & 0.12 & 0.01 & 0.00 \\
\hline & $\mathrm{D}$ & 0.15 & 0.50 & 0.10 & 0.05 & 0.50 & 0.01 & 0.17 & 0.50 & 0.00 \\
\hline \multirow{4}{*}{$63 \%$} & A & 0.03 & 0.02 & 0.01 & 0.00 & 0.02 & 0.00 & 0.00 & 0.02 & 0.00 \\
\hline & $\mathrm{B}$ & 0.05 & 0.04 & 0.01 & 0.02 & 0.04 & 0.00 & 0.01 & 0.04 & 0.00 \\
\hline & $\mathrm{C}$ & 0.20 & 306.73 & 0.13 & 0.05 & 0.22 & 0.03 & 0.06 & 0.04 & 0.00 \\
\hline & $\mathrm{D}$ & 0.20 & 0.48 & 0.13 & 0.15 & 0.48 & 0.09 & 0.15 & 0.37 & 0.00 \\
\hline \multicolumn{2}{|c|}{ Average deviation } & 0.09 & 38.51 & 0.06 & 0.04 & 0.19 & 0.02 & 0.06 & 0.14 & 0.00 \\
\hline
\end{tabular}

With the purpose of maintaining a balance between a random and greedy selection in the GRASP and HOA algorithms, the alpha value chosen was $\alpha=0.5$ for all scenarios. In order to investigate the performance of the proposed methodologies, tests considering time limits of 300,600 , and 1200 s were carried out. Moreover, an additional test considering a relatively large time limit $(4 \mathrm{~h})$ was executed to study the convergence of the proposed methodologies. Table 2 shows the objective function value for each of the PEV charging scenarios for different time limits. In this table, it can be observed that, in most cases, the best objective function values were found using the HOA methodology. For this reason, the information presented about power consumption, minimum voltage, and power losses correspond to the results obtained using the HOA methodology. Note that there was little difference between the three proposed methodologies in terms of the value of the objective function. Table 3 shows the deviation of the objective function (in percentage) found by all methodologies for each PEV charging scenario relative to the best solution found in each case. The last row of the table contains the average deviation percentage for each case. The results show that all values corresponding to the HOA methodology had the smaller average deviation, close to zero, confirming that this methodology provides better solutions for the PEVCC problem. Additionally, Fig. 3 illustrates the improvement on the objective function for each proposed methodology: GRASP, TS, and HOA, with $63 \%$ of PEV penetration for case B. It is possible to observe that good-quality solutions are quickly found, specifically by the GRASP and Hybrid methods.

The power consumption of the system (power generated by the substation and DG units) is shown in Figs. 4 and 5. The red areas represent the consumption of PEVs with priority conditions, the green areas represent the consumption of PEVs without priority conditions, and the white areas represent the conventional loads. As mentioned in Section 3.1.2, the initial solution proposed (uncoordinated charging) had low voltage problems and congestion in the feeders, as well as an increase in energy losses. Figs. 4 and 5 show that, after applying the proposed HOA, the power consumption of the system was modified to eliminate these infeasibilities.

The minimum voltages in each time interval are presented in Figs. 6 and 7. Figs. 6(a) and 7(a) show that for cases A and B, the uncoordinated charging violated the voltage limits in the first five time intervals. A similar situation occurred for Cases $C$ and $D$ related to charging without priority conditions, as shown in Figs. 6(b) and (b). In these cases, the uncoordinated charging violated the voltage limits in the last five time intervals due to the PEVs being charged in the periods with the lowest cost of energy. Note that after the application of the HOA methodology, the solutions found for all cases did not present any operational constraint violations. It is also possible to observe that the voltage profile improved with the use of DG units (Cases B and D).
The total energy losses of the EDS are presented in Fig. 8. The uncoordinated charging of the PEVs led to greater energy losses than with optimized charging. The solution found by the proposed HOA distributed the PEV charging adequately, avoiding an increase in the peak load and achieving a significant reduction in energy
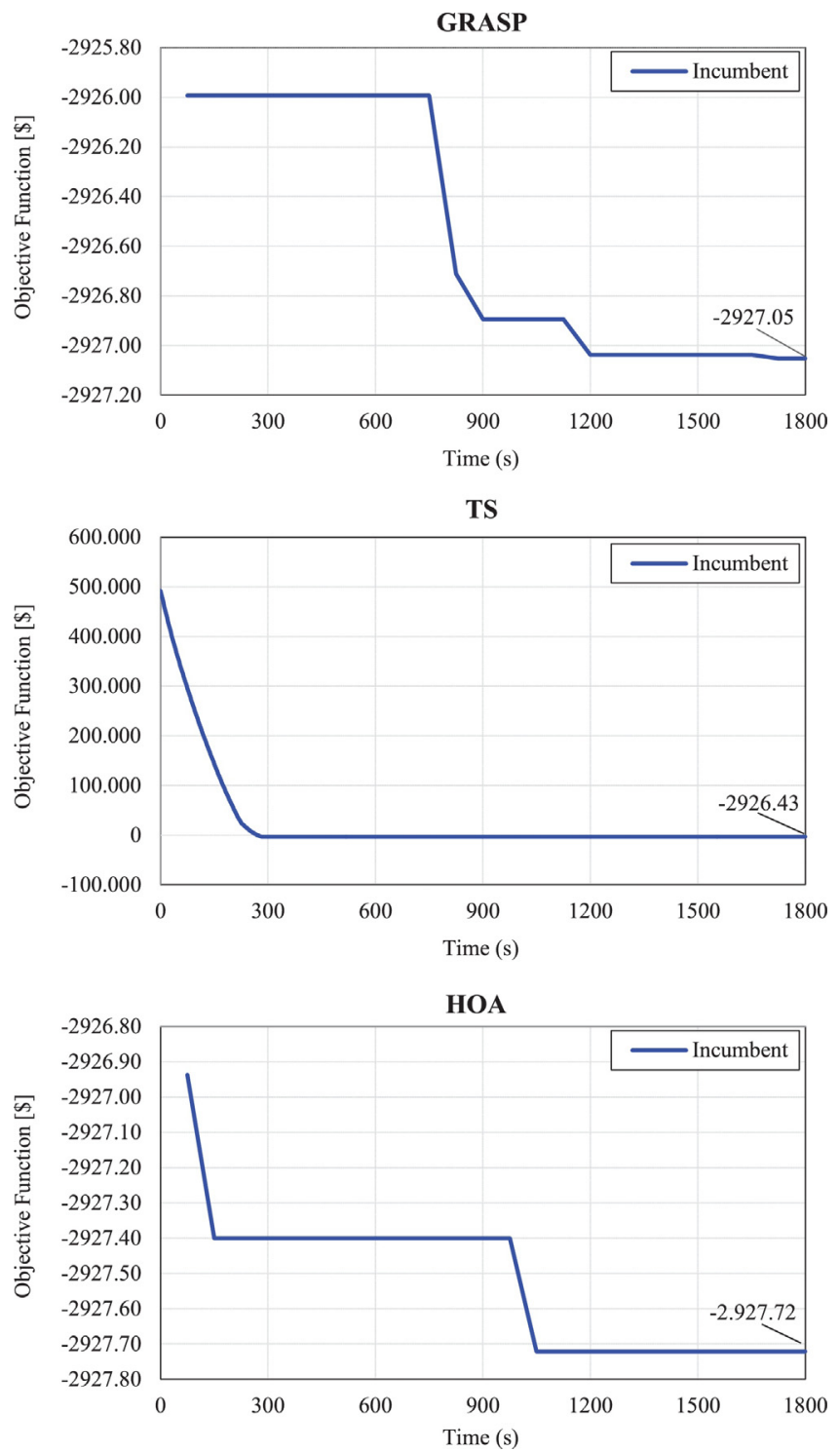

Fig. 3. Convergence of the proposed methodologies during the iterative process for Case B with $63 \%$ PEV penetration. 

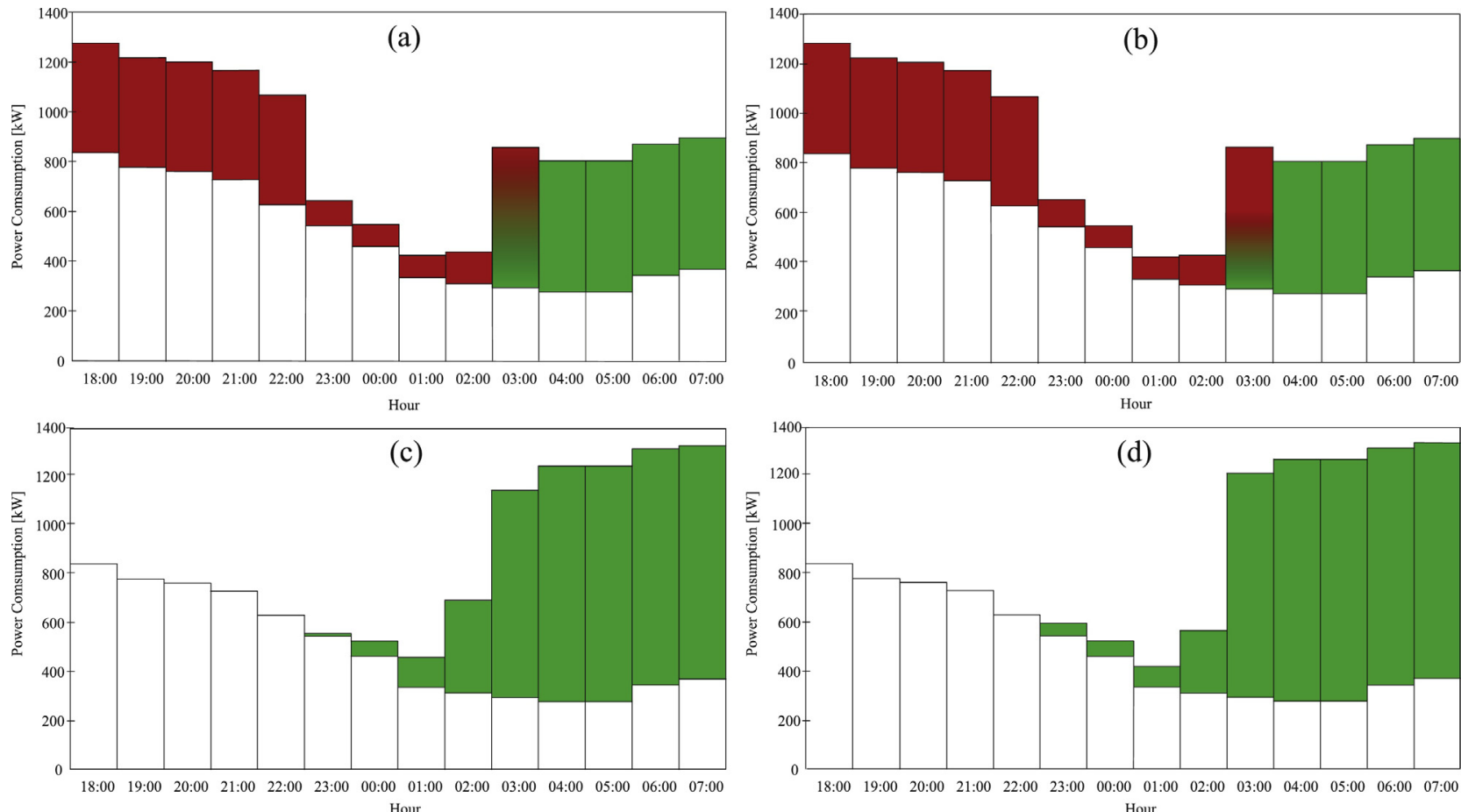

Fig. 4. Power consumption found by the HOA for the tests considering 47\% PEV penetration: (a) Case A. (b) Case B. (c) Case C. (d) Case D.
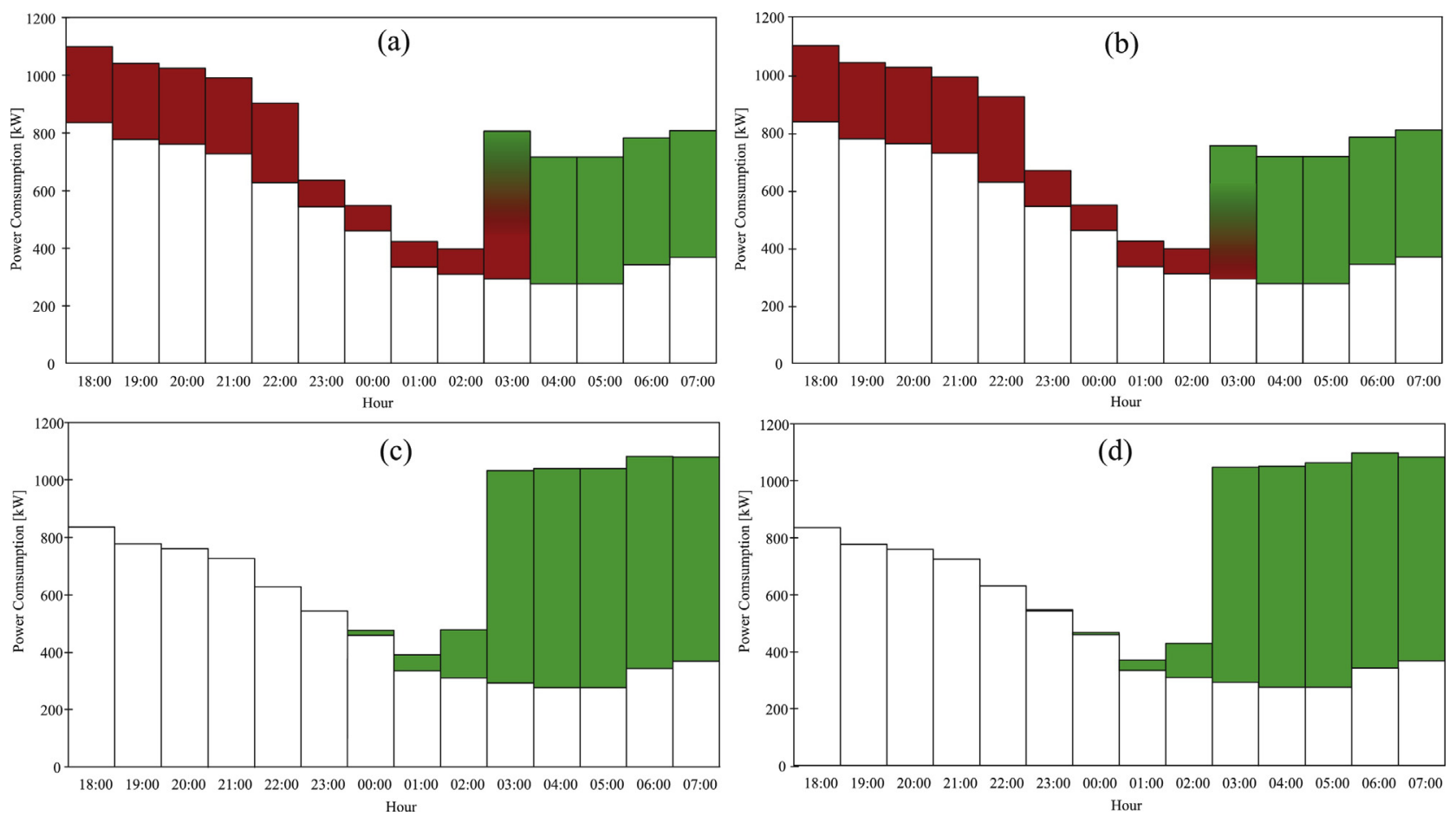

Fig. 5. Power consumption found by the HOA for the tests considering 63\% PEV penetration (a) Case A. (b) Case B. (c) Case C. (d) Case D.

losses. Moreover, note that for Cases B and D, which considered DG units, the value of the total energy losses was smaller compared to the other cases.

Table 4 shows a summary of the best objective functions and related costs for each charging scenario. The proposed HOA improved the operation of the distribution system by reducing the energy losses and eliminating the infeasibilities compared to uncoordinated charging. It is important to highlight the fact that Cases A and B presented negative values for the objective function, due to the term that encourages the quick charging of the
PEVs with priority conditions. In these cases, the penalization cost, represented by (4), is equal to zero, given that the solutions are feasible. Therefore, in the found solutions, both the priority term in (3) and the penalization cost in (4) are minimized in order to improve the objective function. For Cases $C$ and D, the proposed HOA resulted in the charging of the PEV batteries in time intervals with lower energy costs. On the other hand, in Cases $B$ and D considering DG units, the best objective functions were obtained because the cost of the energy generated by the DGs was lower. 
Table 4

Total operational cost of the 449-node system using HOA methodology.

\begin{tabular}{|c|c|c|c|c|c|c|}
\hline Cost $(\$)$ & Uncoordinated charging & A & B & Uncoordinated charging & $\mathrm{C}$ & $\mathrm{D}$ \\
\hline Energy losses & 19.94 & 16.61 & 14.66 & 13.80 & 13.59 & 12.04 \\
\hline Energy supplied $(\beta)$ & 575.71 & 563.11 & 497.22 & 499.33 & 501.00 & 436.26 \\
\hline Priority EV charging $(\gamma)$ & -2793.47 & -2120.38 & -2129.18 & 0.00 & 0.00 & 0.00 \\
\hline Penalizations $(\delta)$ & 28334.13 & 0.00 & 0.00 & 81459.67 & 0.00 & 0.00 \\
\hline Total cost & 26136.30 & -1540.67 & -1617.30 & 81972.79 & 514.59 & 448.30 \\
\hline
\end{tabular}

$63 \%$ - PEV charging

\begin{tabular}{|c|c|c|c|c|c|c|}
\hline $\operatorname{Cost}(\$)$ & Uncoordinated charging & A & B & Uncoordinated charging & C & $\mathrm{D}$ \\
\hline Energy losses & 22.97 & 19.32 & 16.60 & 15.93 & 15.43 & 13.94 \\
\hline Energy supplied $(\beta)$ & 651.13 & 638.45 & 570.76 & 536.55 & 541.15 & 477.34 \\
\hline Priority EV charging $(\gamma)$ & -4190.21 & -3515.10 & -3515.07 & 0.00 & 0.00 & 0.00 \\
\hline Penalizations $(\delta)$ & 687861.37 & 0.00 & 0.00 & 320947.34 & 0.00 & 0.00 \\
\hline Total cost & 684345.26 & -2857.34 & -2927.72 & 321499.82 & 556.58 & 491.28 \\
\hline
\end{tabular}
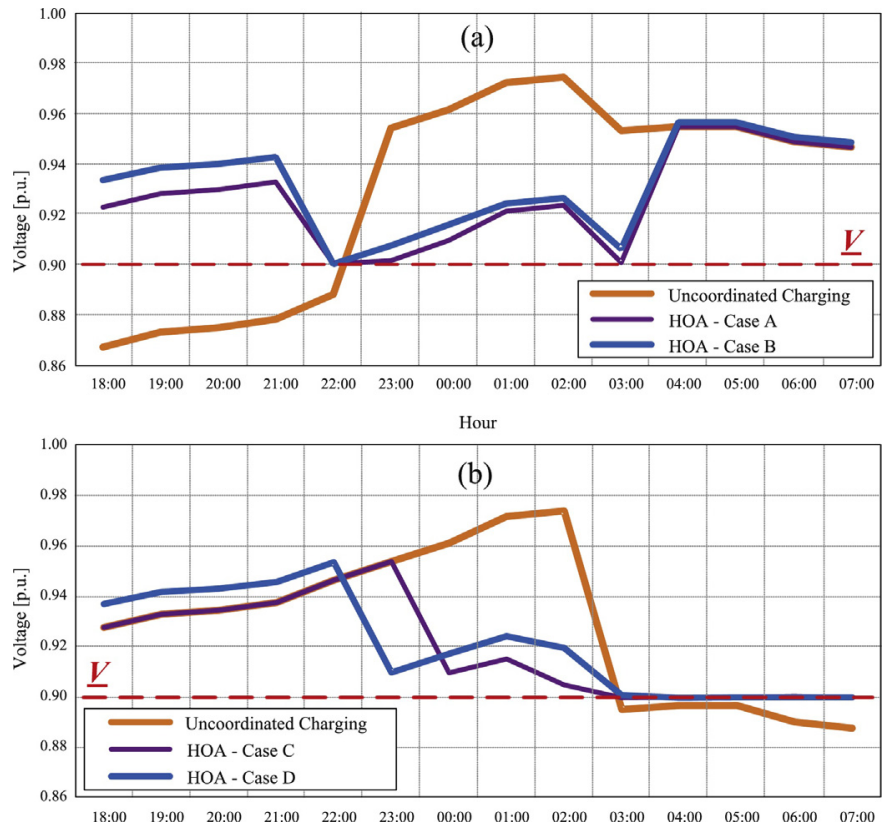

Fig. 6. Minimum voltage for the cases with $47 \% \mathrm{PEV}$ penetration. (a) Cases A and B. (b) Cases C and D.

The power injected by the DG units is shown in Fig. 9. Note that the DG units reached their maximum generation during the first time intervals available for PEV charging. This makes sense because, in these time intervals, the cost of the energy provided
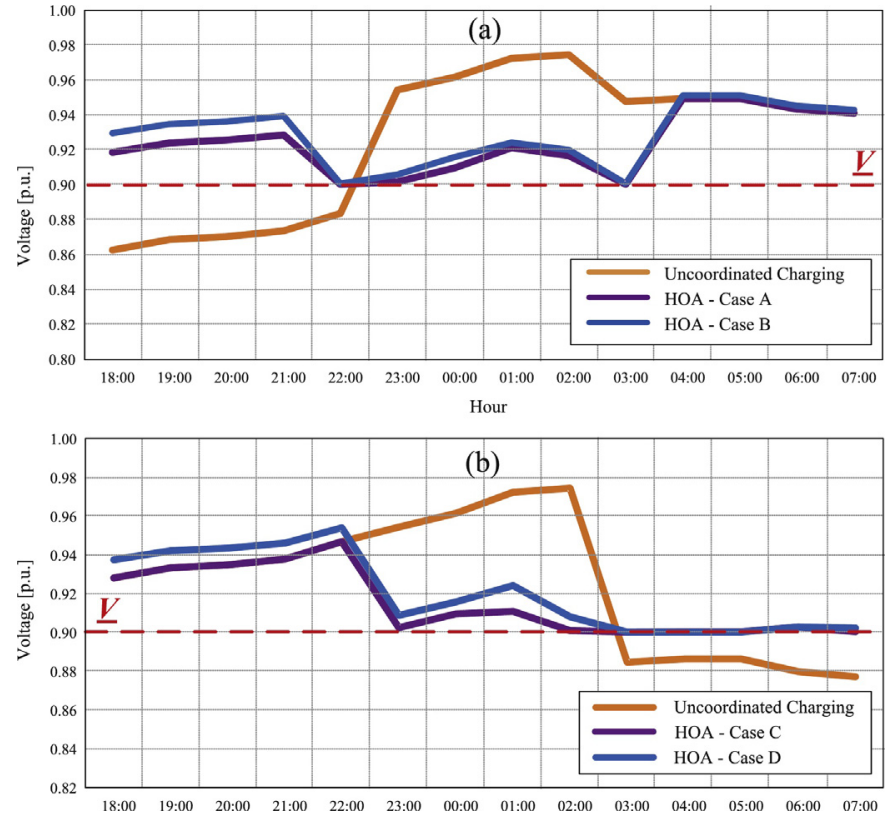

Fig. 7. Minimum voltage for the system with $63 \% \mathrm{PEV}$ penetration. (a) Cases A and B. (b) Cases C and D.

by the substation was greater than that of the energy generated by the DG units. Moreover, for Case B, which considered priority conditions, there was a concentration of the PEV load in the same time intervals taking advantage of the DG operation. In Case D, which did not consider priority, a similar situation occurred, but with the

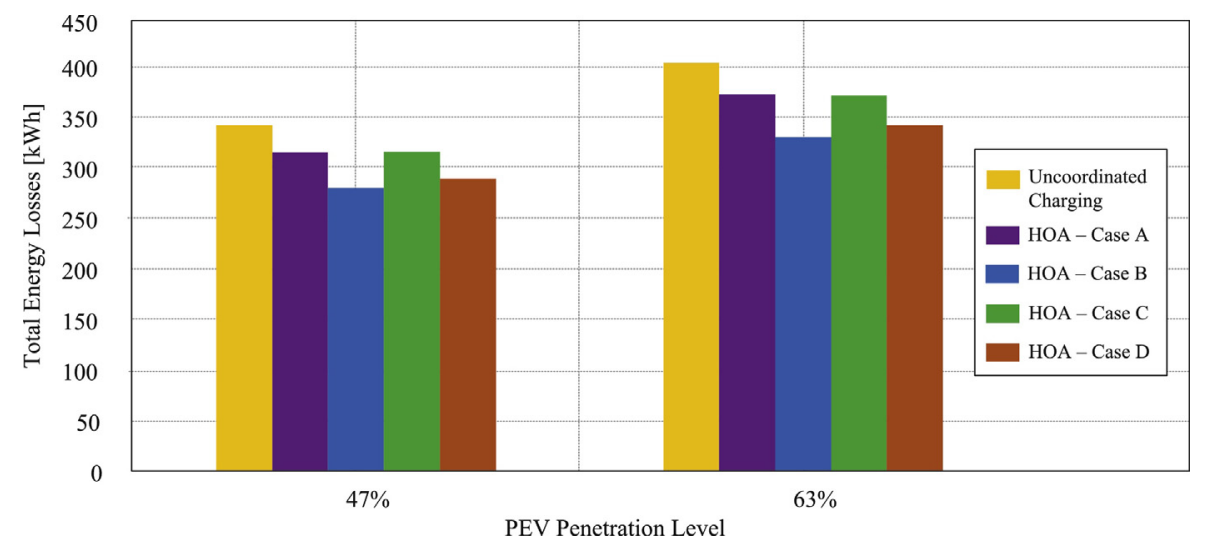

Fig. 8. Total energy losses of the system. 

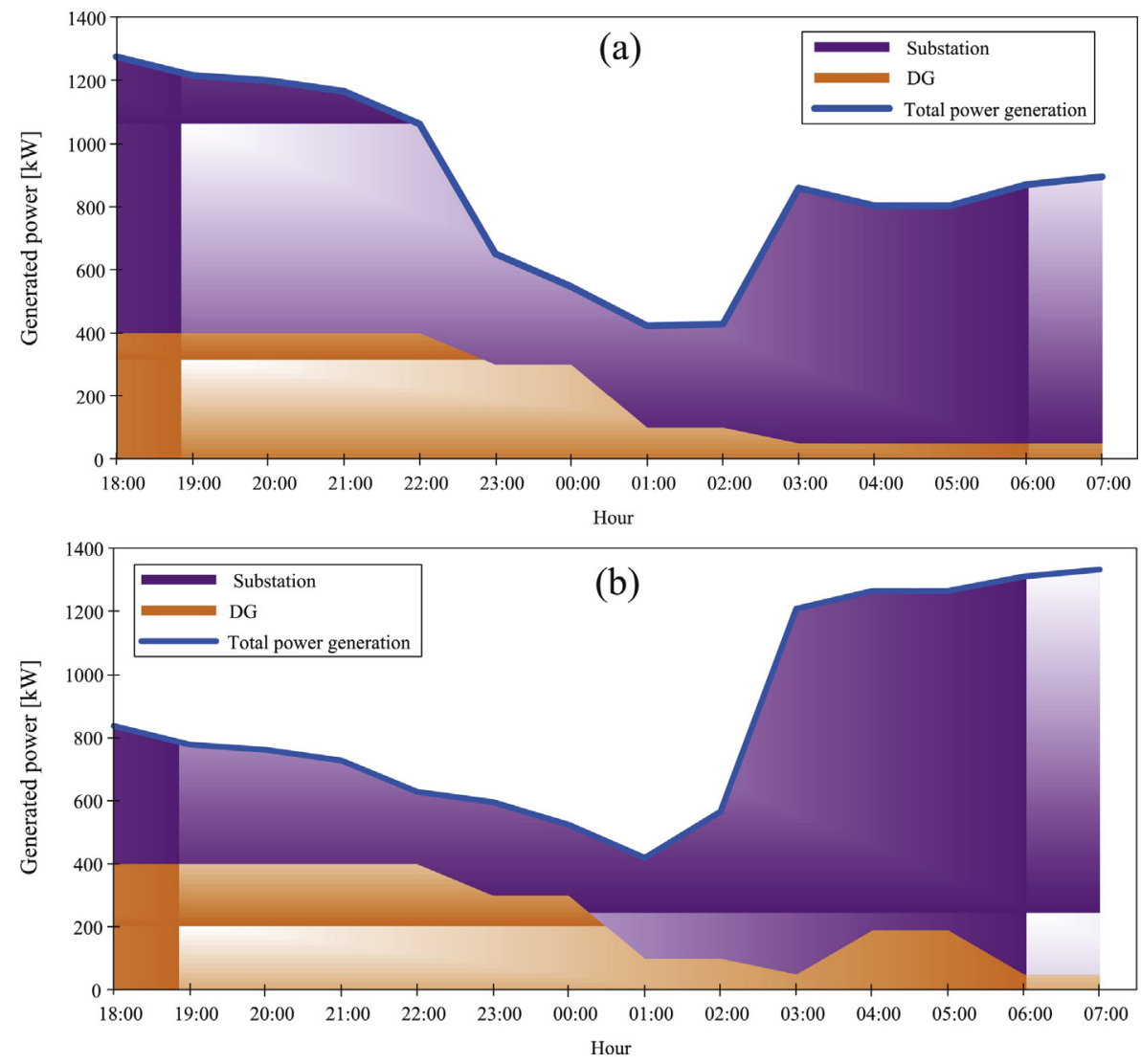

Fig. 9. Generated power with 63\% PEV penetration. (a) Case B. (b) Case D.

difference that the DG units generated more power over the last time intervals due to the concentration of the PEV load in the time intervals with lower energy cost. In this case, the power generated by the DG units contributed eliminating the voltage and current violations, reducing the energy losses, and achieving an efficient system operation.

An additional test was conducted in order to analyze the effectiveness of the proposed HOA. This test was carried out by using case B with 63\% PEV penetration under different conditions for the conventional demand (i.e., conventional demands increased by $15 \%$ ). The algorithm was executed for $1200 \mathrm{~s}$, and the proposed HOA found the best solution, which has a cost equal to -2856.92 . It must be highlighted that this solution is feasible, i.e., it does not present any penalization, despite the fact that the conventional load was increased. Although both the energy losses and the cost of the supplied energy grew with the load increase, the proposed HOA improved the operation of the distribution system and eliminated the infeasibilities in comparison with an uncoordinated charging, as in the previous cases. The tests carried out illustrate the effectiveness and robustness of the proposed method for different scenarios of demand and PEV penetration level.

It must be noted that the results show that the obtained solutions do not present penalizations, i.e., they are feasible. The optimization process seeks the minimization of the objective function, and therefore, solutions with high cost due to penalizations are disregarded in this process.

\section{Conclusions}

Optimization algorithms based on Tabu Search, GRASP, and a novel hybrid optimization algorithm (HOA) were proposed in order to solve the plug-in electric vehicle charging coordination (PEVCC) problem in electrical distribution systems. The results show that the proposed methodologies can be used to obtain the coordinated charging of PEV batteries, while maintaining the economical operation of the distribution system and satisfying the operational and priority constraints.

The three methodologies achieved good-quality solutions for the PEVCC problem compared to the results from an uncoordinated charging scenario. However, after making a comparative analysis between the three proposed methodologies, it was concluded that although all found similar solutions, the HOA provided better results for the solution of the PEVCC problem.

The results demonstrate that the presence of Distributed Generation (DG) units benefits the charging of PEVs. The voltage profile and the energy losses of the system are improved after the application of the proposed methodologies.

The results obtained show that the proposed methodologies provide good-quality solutions for the PEVCC problem in low computational time. Therefore, they can be auxiliary tools in smart grid schemes for the control of future distribution systems.

\section{Acknowledgments}

This work was supported by the Brazilian institutions CAPES, CNPq and FAPESP.

\section{Appendix A.}

The notation used throughout this paper is reproduced below for quick reference. 
Sets

$L \quad$ set of circuits

$N \quad$ set of nodes

$T \quad$ set of time intervals

\section{Parameters}

$\alpha \quad$ grasp parameter

$\beta \quad$ cost of the energy supplied by the substation and DG units [\$/kWh]

$b_{i} \quad$ linear probability distribution function

$\delta \quad$ penalization cost associated to voltage and current limit violations and non-charged PEV energy

$\Delta_{t} \quad$ duration of time interval $t$

$\varepsilon_{j}^{c h} \quad$ efficiency factor of the PEV battery charger

$\eta \quad$ cost of the total energy provided by the DGs $[\$ / \mathrm{kWh}]$

$E_{j}^{v} \quad$ energy that must be charged to the PEV at node $j[\mathrm{~kW}]$

$\varphi \quad$ penalization factor due to unsupplied PEV energy [ $\$ / \mathrm{kWh}]$

$\Phi_{k, t} \quad$ sensitivity index

$\gamma$ component of the objective function to encourage the charging of PEVs

$\bar{I}_{i j} \quad$ current capacity of circuit $i j[\mathrm{~A}]$

$\kappa_{j} \quad$ PEV with charging priority connected at node $j$

$\lambda$ number of time intervals required to charge a PEV

$\mu_{t} \quad$ energy price in time interval $t$

$p_{i} \quad$ probability of choosing an ith element to form the sorted RCL

$P_{i, t}^{d} \quad$ active power demanded at node $i$ in time interval $t[\mathrm{~kW}]$

$\bar{P}_{i}^{g} \quad$ maximum active power of DG unit at node $i[\mathrm{~kW}]$

$\bar{P}^{v} \quad$ maximum power of the PEV batteries $[\mathrm{kW}]$

$p f_{i} \quad$ power factor limit of the DG at node $i$

$Q_{i, t}^{d} \quad$ reactive power demanded at node $\mathrm{i}$ in time interval $t$ [kVAr]

$\bar{Q}_{i}^{g} \quad$ maximum reactive power of DG at node $i$ [kVAr]

$Q_{i}^{g} \quad$ minimum reactive power of DG at node $i$ [kVAr]

$r_{i} \quad$ position of the element $i$ in the sorted RCL

$R_{i j} \quad$ resistance of circuit $i j[\Omega]$

$\rho_{t} \quad$ parameter used to encourage charging with priority $[\$ / \mathrm{kW}]$

SE $\quad$ substation node

$\sigma$ penalization factor due to the current limit violation $\left[\$ / A^{2}\right]$

$S O C_{j}^{i n i} \quad$ initial state of charge of the PEV battery $j$

$\theta$

penalization factor due to the voltage limit violation $\left[\$ / V^{2}\right]$

$\underline{V} \quad$ minimum voltage magnitude $[V]$

$\bar{V} \quad$ maximum voltage magnitude $[V]$

$X_{i j} \quad$ reactance of circuit $i j[\Omega]$

$Z_{i j} \quad$ impedance of circuit $i j[\Omega]$

\section{Variables}

$I_{i j, t} \quad$ current magnitude in circuit $i j$ in time interval $t[\mathrm{~A}]$

$\pi_{i} \quad$ PEVs uncharged energy [kWh]

$P_{i j, t} \quad$ active power flow in circuit $i j$ in time interval $t[\mathrm{~kW}]$

$P_{i, t}^{g} \quad$ active power generated at node $i$ in interval $t[\mathrm{~kW}]$

$P_{i, t}^{v} \quad$ active power demanded by the PEV at node $i$ in time interval $t[\mathrm{~kW}]$

$Q_{i j, t} \quad$ reactive power flow of the circuit $i j$ in time interval $t$ [kVAr]

$Q_{i, t}^{g} \quad$ reactive power generated at node $i$ in time interval $t$ [kVAr]

$V_{i, t} \quad$ voltage magnitude at node $i$ in time interval $t$ [V]

$x_{i, t}^{v} \quad$ binary variable associated with the PEV charging state at node $i$ in time interval $t$

\section{References}

[1] A. Ipakchi, F. Albuyeh, Grid of the future, Power Energy Mag. 7 (March-April) (2009) 52-62.

[2] W. Kempton, V. Udo, K. Huber, K. Komara, S. Letendre, S. Baker, D. Brunner, N Pearre, A Test of Vehicle-to-Grid (V2G) for Energy Storage and Frequency Regulation in the PJM System, 2008, Newark, DE.

[3] IEA, Global EV Outlook: Understanding the Electric Vehicle Landscape to 2020, OECD/IEA, Paris, 2013, April.

[4] J.A.P. Lopes, F.J. Soares, P.M.R. Almeida, Integration of electric vehicles in the electric power system, Proc. IEEE 99 (January (1)) (2011) 168-183.

[5] S. Lin, Z. He, T. Zang, Q. Qian, Impact of plug-in hybrid electric vehicles on distribution systems, in: Int. Conf. Power Syst. Tech. (POWERCON), 24-28 October, 2010, pp. 1-5

[6] K. Clement-Nyns, E. Haesen, J. Driesen, The impact of charging plug-in hybrid electric vehicles on a residential distribution grid, IEEE Trans. Power Syst. 25 (February (1)) (2010) 371-380.

[7] E. Sortomme, M.M. Hindi, S.D.J.M. Pherson, S.S. Venkata, Coordinated charging of plug-in hybrid electric vehicles to minimize distribution system losses, IEEE Trans. Smart Grid 2 (March (1)) (2011) 198-205.

[8] C. Jin, J. Tang, P. Ghosh, Optimizing electric vehicle charging with energy storage in the electricity market, IEEE Trans. Smart Grid 4 (1)(2013, March) 311-320.

[9] Z. Luo, Z. Hu, Y. Song, Z. Xu, H. Lu, Optimal coordination of plug-in electric vehicles in power grids with cost-benefit analysis-Part I: enabling techniques, IEEE Trans. Power Syst. 28 (November (4)) (2013) 3546-3555.

[10] S. Deilami, A.S. Masoum, P.S. Moses, M.A.S. Masoum, Real-time coordination of plug-in electric vehicle charging in smart grids to minimize power losses and improve voltage profile, IEEE Trans. Smart Grid 2 (September (3)) (2011) 456-467.

[11] O. Sundstroem, C. Binding, Flexible charging optimization for electric vehicles considering distribution grid constraints, IEEE Trans. Smart Grid 3 (March (1)) (2012) 26-37.

[12] K. Clement, E. Haesen, J. Driesen, Coordinated charging of multiple plug-in hybrid electric vehicles in residential distribution grids, in: Proc. Power Syst. Conf. Expo., 2009, pp. 1-7.

[13] Lunci Hua, Jia Wang, Chi Zhou, Adaptive electric vehicle charging coordination on distribution network, IEEE Trans. Smart Grid 5 (6) (2014 Nov) 2666-2675

[14] Zhongjing Ma, D.S. Callaway, I.A. Hiskens, Decentralized charging control of large populations of plug-in electric vehicles, IEEE Trans. Control Syst. Technol. 21 (January (1)) (2013) 67-78.

[15] W. Chao-Kai, C. Jung-Chieh, T. Jen-Hao, T. Pangan, Decentralized plug-in electric vehicle charging selection algorithm in power systems, IEEE Trans. Smart Grid 3 (December (4)) (2012) 1779-1789.

[16] S. Hutterer, F. Auinger, M. Affenzeller, Metaheuristic optimization of electric vehicle charging strategies in uncertain environment, in: Int. Conf. Probabilistic Methods Applied to Power Systems, 2012

[17] J. Yang, L. He, S. Fu, An improved PSO-based charging strategy of electric vehicles in electrical distribution grid, Appl. Energy 128 (September) (2014) $82-92$.

[18] A. Masoum, S. Deilami, A. Abu-Siada, M. Masoum, Fuzzy approach for online coordination of plug-in electric vehicle charging in smart grid, IEEE Trans. Sustain. Energy 6 (July (3)) (2015) 1112-1121.

[19] S.J. Gunter, D.J. Perreault, S. Suresh, K.K. Afridi, Methodology for the optimal design of PEV charging systems with multiple chargers and distributed resources, IEEE PES Innovative Smart Grid Technologies (ISGT), 24-27 February (2013) 1-6.

[20] S.Y. Derakhshandeh, A.S. Masoum, S. Deilami, M.A.S. Masoum, M.E. Hamedani Golshan, Coordination of generation scheduling with PEVs charging in industrial microgrids, IEEE Trans. Power Syst. 28 (August (3)) (2013) 3451-3461

[21] S. Shengnan, F. Jahanbakhsh, J.R. Aguero, Le Xu, Integration of PEVS and PV-DG in power distribution systems using distributed energy storage dynamic analyses, in: IEEE PES Innovative Smart Grid Technologies (ISGT), 24-27 February, 2013, pp. 1-6.

[22] J.M. Foster, G. Trevino, M. Kuss, M.C. Caramanis, Plug-in electric vehicle and voltage support for distributed solar: theory and application, IEEE Syst. J. 7 (December (4)) (2013) 881-888.

[23] H. Weihao, S. Chi, C. Zhe, B. Bak-Jensen, Optimal operation of plug-in electric vehicles in power systems with high wind power penetrations, IEEE Trans. Sustain. Energy 4 (July (3)) (2013) 577-585.

[24] W. Ting, Y. Qiang, B. Zhejing, Y. Wenjun, Coordinated energy dispatching in microgrid with wind power generation and PEVS, IEEE Trans. Smart Grid 4 (September (3)) (2013) 1453-1463.

[25] P. Papadopoulos, S. Skarvelis-Kazakos, I. Grau, L.M. Cipcigan, N. Jenkins, Predicting electric vehicle impacts on residential distribution networks with distributed generation, in: Proc. IEEE Veh. Power Propulsion Conf. (VPPC), 1-3 September, 2010, pp. 1-5.

[26] T. Sousa, H. Morais, Z. Vale, P. Faria, J. Soares, Intelligent energy resource management considering vehicle-to-grid: a simulated annealing approach, in: IEEE Pow. \& Energy Society Gen. Meeting, 22-26 July, 2012.

[27] F. Glover, M. Laguna, Tabu Search, Kluwer Academic Publishers, Norwell, 1997.

[28] T.A. Feo, M.G.C. Resende, Greedy randomized adaptive search procedures, J. Glob. Optim. 6 (2) (1995) 109-133. 
[29] M.D. Galus, R.A. Waraich, G. Andersson, Predictive, distributed, hierarchical charging control of PHEVs in the distribution system of a large urban area incorporating a multi agent transportation simulation, in: 17th Power Syst. Computation Conf., Stockholm, Sweden, 2011.

[30] J.F. Franco, M.J. Rider, R. Romero, An MILP model for the plug-in electric vehicle charging coordination problem in electrical distribution systems, in: IEEE Pow. \& Energy Society Gen. Meeting, 27-31 July, 2014.

[31] R.R. Gonçalves, R.P. Alves, J.F. Franco, M.J. Rider, Operation planning of electrical systems using a mixed integer linear model, J. Control Autom. Electr. Syst. 24 (June (3)) (2013)
[32] R.G. Cespedes, New method for the analysis of distribution networks, IEEE Trans. Power Deliv. 5 (January (1)) (1990) 391-396.

[33] Nissan Vehicles - Model Specifications. Available at: http://www.nissanusa. com/electric-cars/leaf/versions-pecs/version.sl.html.

[34] T. Frierich, J. He, N. Hebbinghaus, F. Neumann, C. Witt, Analyses of simple hybrid algorithms for the vertex cover problem, in: 14th IEEE Congress on Evolutionary Computation, Piscataway, 2007, pp. 2614-2621.

[35] G. Pepermans, J. Driesen, D. Haeseldonckx, R. Belmans, W. D’haeseleer,

Distributed generation: definition, benefits and issues, Energy Policy 33 (April 6)) (2005) 787-798 